\title{
How Pandas Shaped China's Approach Towards Foreign Affairs: Economically and Politically
}

\author{
Guo Yin* \\ Wilbraham and Monson Academy, Wilbraham, MA, US \\ *Corresponding author: gyin@wma.us
}

\begin{abstract}
The concept of Panda Diplomacy would be thoroughly explained in two lenses, economics and politics. Pandas, as the cutest ambassadors of China, have served their purpose in both over centuries. As one would know, Pandas are extremely scarce and therefore preserved carefully. Nevertheless, it demonstrates significant political influence since they are only found in China and can be utilized as a tool to improve foreign relations. Early methods like gifting pandas used to be popular until China decided to create a monopoly on the ownership of such favored creatures. Nowadays, giving other countries temporary ownerships of pandas would still create this situation while improving national relations, economic and political benefits. This advantage of permanent ownership of pandas brought long-lasting treaties, peacekeeping cooperation, and income that would positively affect both sides. This symbol indicates a next-level relationship between China and another country that will boost interactions from both, establishing a positive reputation of China's friendly diplomatic approaches and leverage in this relationship.
\end{abstract}

Keywords: Foreign Affairs; Panda Diplomacy; Economics; Politics.

\section{Introduction}

\subsection{Research Background}

Pandas shaped one of the earliest monopolies in Chinese diplomacy. Dating back to the Tang Dynasty, when a pair of bears (presumed as pandas) were sent out to Japan [5] as a symbol of peace. Since then, China has valued the rarity of pandas and remained its living and reproduction quarters mostly in China, regardless of cases when they are rented to other countries. In the past century, China increased the price of renting pandas significantly in many forms, both monetary and political values, due to its increasing mortality rates in foreign states [Figure 1]. In addition to its initial approach of absolute ownership to all giant pandas, lending a panda became harder as stricter regulations were enacted. Such as "giant pandas borrowed should be commensurate with the domestication and breeding of giant pandas in funds, facilities, and personnel and other conditions, achieved with the domestication and breeding of wildlife under special state protection of the giant panda species license" [8]. 

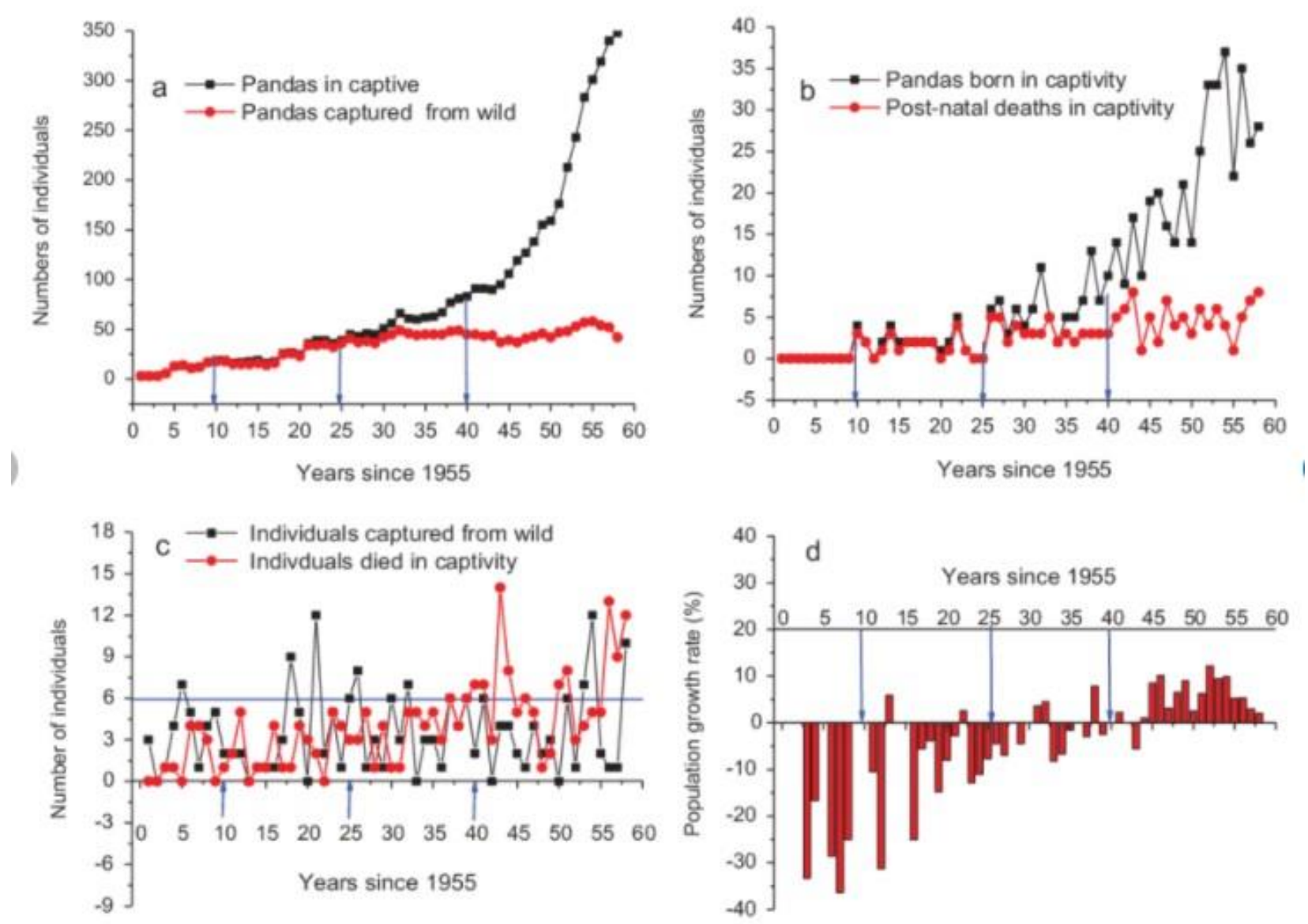

Figure 1. Graphs on the rising mortality rates on Pandas. Adapted from ResearchGate.

\subsection{Literature Review}

Panda diplomacy is widely accepted and recognized as the act of lending giant pandas to other countries and a form of "soft power." Soft power is generally defined as "the ability to affect others to obtain the outcomes one wants through attraction rather than coercion or payment" [7], and pandas became a major method of achieving such a form of advantage. However, there are debates on whether this method of diplomacy of effect the population and preserves giant pandas [4]. For example, Lin's 2009 article claimed that China's panda diplomacy did not contribute to the conservancy of pandas while others debated otherwise.

\subsection{Research Framework}

The impact of panda diplomacy politically on the international level will be explained. The second section would analyze its economic outcomes, using economic models through a variety of perspectives. And finally presenting positive and negative effects of panda diplomacy.

\section{Analytics}

Most parts of this study are based on secondary resources, as it is a more economic-political orientated subject in which personal accounts are inefficient. Also, a smaller portion of historical analysis is used. The development of panda diplomacy will be discussed chronologically, of its early, developing, and matured phases. Economic models used would be included in the final section since earlier data in the first and second phases cannot be accounted for. In return, the first two phases would be backed with historical analysis.

From the beginning, China's methods are relatively generous in terms of giving away pandas since the importance of those fluffy bears is not raised yet. However, China still is testing different 
approaches to gifting and eventually decides to create a monopoly of this speciality. Some policies were established through mostly are treaties of goods or services provided by the country that receives pandas, rather than preserving and caring for pandas, and it was not considered endangered during the early phase.

In the second phase, China began to realize the significance of pandas politically and their scarcity. As a result, regulations began to shift towards the treatment and ownership of pandas. China is using pandas as a tool to maintain friendly relationships for trade and allies since during this period, China lacks resources in many fields.

Finally, by the giant panda's successful maintenance and friendly image, China achieved economic and political success, and pandas are used as significant indicators of friendship, as they are relatively less important to China. Nonetheless, they still bring a boost to local tourism and can make a difference in some countries. With established economic and political influence, China once more restricts regulations on preserving and living conditions of pandas and takes full advantage of giant pandas.

\section{Findings}

\subsection{The Three Phases of Panda Diplomacy}

\subsubsection{Phase One: Realization of the Concept}

China's long history of leasing pandas did not first occur to the Chinese as a key factor of foreign relations until they knew how scarce pandas were. However, the interest in pandas soon grew and changed the value of pandas, which are sent out as gifts on the rarest occasions. This growth in demand certainly gained a bargaining chip for the Chinese and gained international attention to both the cute bears and China.

\subsubsection{Phase Two: Application of Panda Diplomacy}

Since the 1950s, the PRC has continued to use the gifting of pandas as a political strategy to maintain positive relations with friendly countries. For example, two pandas arrived in the US on April 16, 1972, marking a new start of Chinese-American diplomacy after Nixon returned from a visit to China in February. In return, two musk oxen were sent to China later that year [10]. "Eight thousand people stood in the rain for a half-hour or longer to watch the pandas when they first went on display, and an estimated 3 million people a year come to see them" [7].

\subsubsection{Phase Three: Start of Preserving}

The gifting of giant pandas ended in 1982 when China responded to the protection of endangered species and marked an end for traditional gifting and a start for leasing pandas. For China, this means income and an opportunity for workers to travel overseas, which is uncommon in China in the 80s. Also aiming to solve issues regarding pandas in other countries, the China Wildlife Conservation Association and the Chinese Association of Zoological Garde added a series of regulations. Including that all offspring of pandas born outside of China and bodies of dead pandas all belong to China. In addition, programs are launched to manually reproduce pandas with cooperation from five national parks worldwide, with a range of 30 pandas [5].

\subsection{Economic Model: PESTEL}

\subsubsection{Political}

Pandas are described as strong but yet peaceful and friendly creatures, known for their gentle temperament and not attacking others [10]. The Yin and Yang represented by the black and white colors of the panda demonstrate harmony and peace, showing China's attitude toward its foreign policies. 


\subsubsection{Economical}

China had established multiple foreign trade deals through the gifting or lending of pandas successfully. In early 2011, China established multi-billion-dollar agreements with Scotland to supply salmon and petrochemical and renewable energy tech; and China loaned a pair of pandas to the Edinburgh Zoo [10].

\subsubsection{Social}

Social effects would include a national boost in tourism, leading to closer interactions between the people from the two countries, as one learns another's culture demonstrated through pandas. Also, in China, this would increase tourism to that other country since the transfer of pandas would bring more recognition to that country and encourage travel or purchase of goods of that country.

\subsubsection{Technological}

Cooperative efforts of preserving and reproducing pandas have significantly reduced the decreasing rate of the panda population and infant mortality rate. Shortly, pandas will no longer be considered endangered.

\subsubsection{Environmental}

Renting pandas would not only increase awareness on preserving pandas but also other endangered species. Tourists would realize how a species of animal would easily be eradicated by human activity. Similar ideals inspired movements regarding sea turtles and an improvement of recycling plastics to avoid micro plastics in the ecosystem [6].

\subsubsection{Legal}

China makes all regulations on pandas, and to borrow a panda, a country must agree to those terms. Including security, healthcare, diet restrictions, and fees of leasing.

\section{Discussion}

Although the importance of tourism becomes greater, how long would the public be interested in pandas? Pandas are valued more as a political symbol rather than an exotic animal nowadays, and people would eventually be no longer interested in them. To provide sustainability towards panda diplomacy, China should aim to accomplish the following:

\subsection{Extending or Reducing the Least Amount of Time for Leasing}

By extending the initial leasing time, China would gain more money to start with, and optimistically, extend the end period. However, there is a chance that this method might backfire, exceeding the price over the demand over the equilibrium [Figure 2]. Another approach would be to make pandas remain scarce, or even more scarce so that there would always be countries looking to rent a panda so that the influence of pandas would remain. The economic incentives are not the most appealing towards China [Figure 3]. 


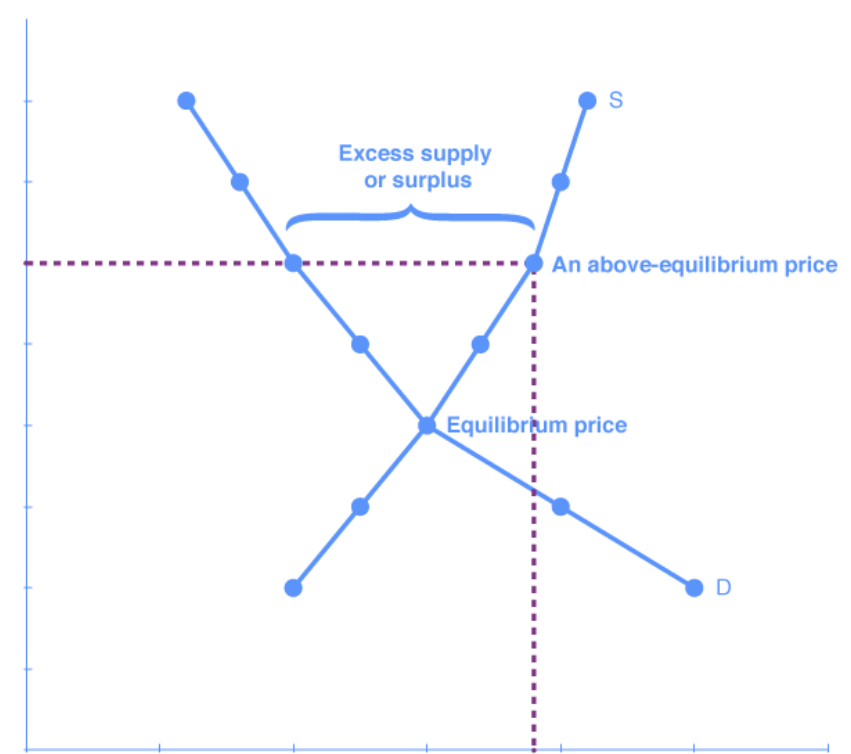

Figure 2. Graph explaining the number of countries willing to rent pandas above the equilibrium, creating a surplus of renters. Adopted from Lumen, "Equilibrium, Surplus, and Shortage".

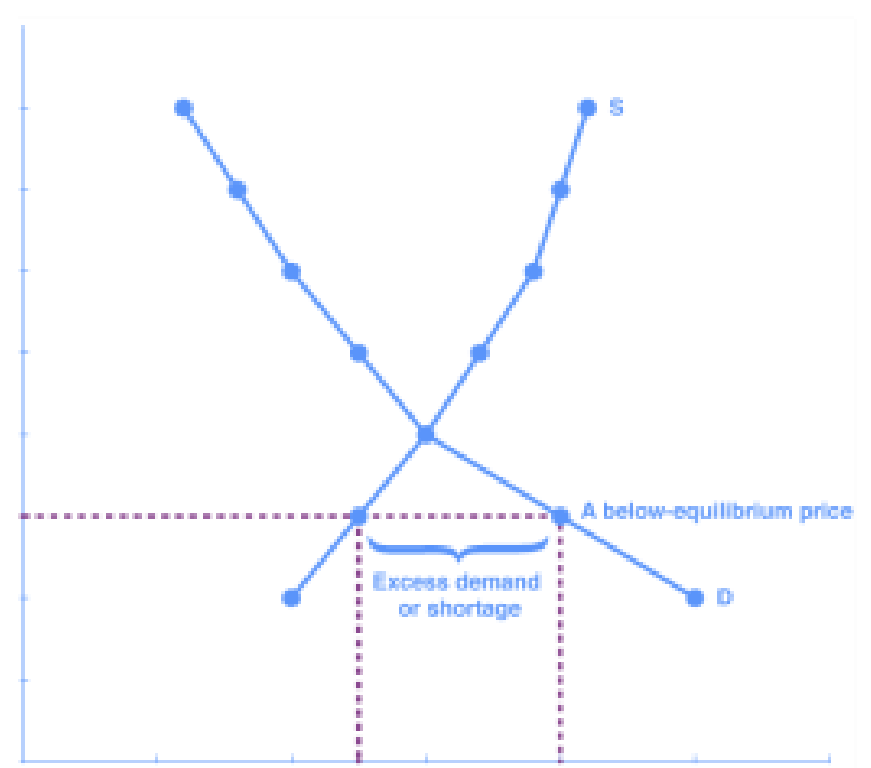

Figure 3. Graph explaining the shortage of pandas, how there is not enough pandas to satisfy the demand of countries willing to rent them. Adopted from Lumen, "Equilibrium, Surplus, and Shortage".

\subsection{Assimilation of Cultures}

After all, the use of pandas is more than a simple gift. But it also educates people about the Chinese culture. Instead of educating tourists about pandas, introducing the Chinese culture's concept of Yin and Yang would bring them further from simply observing a unique animal. The philosophy of panda diplomacy can be applied to a wide range of subjects besides pandas. Chinese calligraphy, for example, has the same characteristics as pandas: it only exists in China, the creator has control of its price, and it's a significant part of history. Perhaps tourists that were educated about Chinese history would be interested in calligraphy, and another monopoly would be established, extending from pandas from a mostly national level to a local or regional focus. 


\section{Conclusion}

With pandas, China achieved success politically and successfully through the management of trade, preserving in pandas, and foreign relations. Efforts of panda diplomacy are demonstrated in five perspectives; PESTEL (political, economic, social, technological, environmental, and legal). However, panda diplomacy mainly affects politically and economically. And this method can be extended by China through either increasing or reducing the least amount of time renting pandas or using another medium of Chinese culture like calligraphy that China is dominant in.

\subsection{Significance}

The significance of panda diplomacy is shown through historical, economic, and political analysis. It is a spectacular method of generating revenue and maintaining foreign relations for both sides. In addition, it is an important part of Chinese culture.

\subsection{Limitations and Future Studies}

Though historical analysis is relatively accountable, it still does not correlate with demographics and economic improvements during phase one of the development of panda diplomacy. More research on the historical recording of panda-gifting would be essential, though there is very little mentioning of this particular method of diplomacy centuries ago.

\section{Reference}

[1] BaiduBaike.(n.d.).PandaDiplomacyhttps://baike.Baidu.com/item/\%E7\%86\%8A\%E7\%8C\%AB\%E5\%A 4\%96\%E4\%BA\%A4 / 2419786.

[2] Burns, A. (2016, February 4). When Ling-Ling and Hsing Hsing arrived in the U.S. The New York Times. https: //www.nytimes.com/ 2016/ 02/07/ nyregion/ the-pandas-richard-nixon-obtained-for-the-us.html

[3] Cohn, D., \& Masters, B. (1992, December 31). Ling-Ling Dies Suddenly. The Washington Post. Https: // www. washingtonpost.com/ wp-srv/ local/ daily/ june99/ lingdies92.htm

[4] Lin Wen-cheng. (2009). China's Panda Diplomacy. Institute of China and Asia Pacific Studies.

[5] Holland, B. (2017, March 16). Panda diplomacy: The world's cutest ambassadors. HISTORY. https:// www. History. com/ news/ panda-diplomacy-the-worlds-cutest-ambassadors

[6] National Ocean Service. (2021, January 26). What are micro plastics? NOAA. Https: // oceanservice. noaa.gov/facts/microplastics.html\#: :text=Microplastics\%20are\%20small\%20plastic\%20pieces,our\%20 ocean\%20and $\% 20$ aquatic $\% 20$ life.\&text=Plastic\%20debris\%20can\%20come\%20in,)\%20are\%20called $\% 20 \%$ E2\%80\%9Cmicroplastics.\%E2\%80\%9D.

[7] Nye, J. S. (2019). Soft Power and Public Diplomacy Revisited. The Hague Jornal of Diplomacy, 14(1-2). 7-20. Leiden | Boston: Brill | Niihoff

[8] State Forestry Administration. (2011, July 25). Machine translation of "Domestic giant panda loan regulations"(China).Global-Regulation. Https: // www.global- regulation. Com/ translation/ china/ 159669/ domestic-giant-panda-loan-regulations.html.

[9] A. (2017, May 25). The significance of the panda. The Daily China. https://www.thedailychina.org/thesignificance-of-the-panda/.

[10]S. (2013, October 14).China swaps pandas for uranium in tradedeals. CNNMoney. https: // money.cnn.com/ 2013/ 10/ 14/ news/economy/ china-panda-trade/ 